\title{
Evidence for the contribution of genetic variations in regulator of $G$ protein signaling 9 to the genetic susceptibility of heroin dependence
}

\author{
YONGSHENG $\mathrm{ZHU}^{1-3}$ and HONGBO ZHANG ${ }^{1,2}$ \\ ${ }^{1}$ College of Forensic Science, Xi'an Jiaotong University, Key Laboratory of Ministry of Public Health for Forensic Science, \\ Xi'an, Shaanxi 710061; ${ }^{2}$ Key Laboratory of Environment and Genes Related to Diseases, Xi'an Jiaotong University, \\ Ministry of Education, Xi'an, Shaanxi 710061; ${ }^{3}$ Department of Medical Genetics and Cell Biology, \\ Ningxia Medical University, Key Laboratory of Fertility Preservation and Maintenance, \\ Ministry of Education, Ningxia, Yinchuan 750004, P.R. China
}

Received February 11, 2014; Accepted December 19, 2014

DOI: $10.3892 / \mathrm{mmr} .2015 .3210$

\begin{abstract}
Regulator of G protein signaling (RGS) proteins are responsible for the rapid turnoff of $\mathrm{G}$ protein-coupled receptor signaling pathways. RGS9-2, a brain-specific splice variant of the RGS9 gene, is highly expressed in the striatum but lowly expressed in the periaqueductal gray and spinal cord, which mediate various actions of morphine and other opiates. In order to identify the markers that contribute to the genetic susceptibility of heroin dependence, the potential association between heroin dependence and 10 single nucleotide polymorphisms (SNPs), including rs8077696, rs8070231, rs2292593, rs2292592, rs9916525, rs1122079, rs4790953, rs1530351, rs4791230 and rs2869577 of the RGS9 gene was evaluated using the MassARRAY system. The present study recruited 425 heroin-dependent patients and 205 healthy controls. The results revealed that two SNPs (rs1530351 and rs4791230) located in the promoter region of the RGS9 gene, were significantly associated with heroin dependence $(\mathrm{P}<0.05)$. The frequency of the $\mathrm{C}$ allele in $\mathrm{rs} 1530351\left(\chi^{2}=8.031, \mathrm{P}=0.005\right.$, $\mathrm{OR}=2.079,95 \% \mathrm{CI}=1.241-3.483)$ and the $\mathrm{G}$ allele in $\mathrm{rs} 4791230$ $\left(\chi^{2}=7.360, \mathrm{P}=0.007, \mathrm{OR}=2.021,95 \% \mathrm{CI}=1.205-3.389\right)$ in the heroin-dependent patients was significantly higher than that in the controls. Furthermore, linkage disequilibrium was observed in three blocks ( $\left.\mathrm{D}^{\prime}>0.9\right)$ and significantly less T-A haplotypes $\left(\chi^{2}=4.867, \mathrm{P}=0.027, \mathrm{OR}=0.442,95 \% \mathrm{CI}=0.210-0.929\right)$ were identified in the heroin-dependent patients, suggesting that they may exhibit protective effects against heroin dependence.
\end{abstract}

Correspondence to: Dr Hongbo Zhang, College of Forensic Science, Xi'an Jiaotong University, Key Laboratory of Ministry of Public Health for Forensic Science, 76 Yanta West Road, Xi'an, Shaanxi 710061, P.R. China

E-mail: zhanghb@mail.xjtu.edu.cn

Key words: regulators of $\mathrm{G}$ protein signaling, single nucleotide polymorphisms, heroin dependence
These findings indicate a role for RGS9 gene polymorphisms in heroin dependence and may be informative for future genetic or biological studies on heroin dependence.

\section{Introduction}

Drug dependence, generally defined as compulsive drug use and loss of control over drug intake, is induced by repeated exposure to substances, including opioids and psychostimulants $(1,2)$. A previous study demonstrated that there is a genetic component for the risk of substance dependence, particularly opioid dependence (3). Consistently, family and twin studies have demonstrated a substantial genetic effect on the development of drug dependence, with inherited risk estimates in the range of $40-60 \%(4,5)$. A previous study revealed that the regulator of $\mathrm{G}$ protein signaling 9 (RGS9) gene may be associated with drug dependence, including opiate dependence (6).

Human and animal studies revealed that the D2-like dopamine receptors are critical in the development of substance dependence $(7,8)$. The heterotrimeric $\mathrm{G}$ protein system regulates the activity of the dopamine receptors. RGS regulates the function of heterotrimeric $G$ protein in part by stimulating the GTPase activity of the G protein subunits $(9,10)$. RGS9, a member of the RGS family, has two splice isoforms $(9,10)$. The short splice isoform RGS9-1, which contains 18 amino acid residues at the C-terminus is expressed in the photoreceptors of the retina. In the long splice isoform, RGS9-2, the short C-terminus is replaced by a longer region of 209 amino acids (11). RGS9-2 is specifically expressed in the striatum important for opiate reward, but is lowly expressed in the periaqueductal gray and spinal cord important for opiate analgesia (12). Therefore, RGS9-2 has been regarded as a potential partner for $\mathrm{G}$ protein-coupled receptors involved in drug dependence, including morphine (12-14).

The RGS9 gene (17q21-25, $1.1 \mathrm{Mb}$ ) has 18 exons with intron-exon junctions conforming to splice-site consensus sequences (15). Linkage studies demonstrated that the region was implicated in susceptibility to major mental illness (16-19). A single nucleotide polymorphism (SNP) rs4790953 in the 
3'-flanking region of the RGS9 gene was reported to be weakly associated with antipsychotic-induced tardive dyskinesia in a Chinese population in Taiwan (17). However, to the best of our knowledge, the association between the RGS9 gene and heroin dependence has not yet been investigated. In view of the crucial role of RGS9 in heroin dependence, the present study investigated 10 SNPs (rs8077696, rs8070231, rs2292593, rs2292592, rs9916525, rs1122079, rs4790953, rs1530351, rs4791230 and rs2869577) in a Chinese population to verify the putative association between RGS9 polymorphisms and heroin dependence.

\section{Subjects and methods}

Subjects. A total of 425 unrelated heroin-dependent patients (mean age: $35.76 \pm 6.16$ years, 345 males, 80 females) were recruited from the Methadone Maintenance Treatment program of Xi'an Mental Health Center (Xi'an, China). Participants were daily or almost daily users of heroin for a minimum of one year prior to assessment. Their dependence status, major central nervous system (CNS) diseases and psychoses were evaluated by a senior psychiatrist at the beginning of the methadone management program. The diagnosis of opioid dependence was based on the Diagnostic and Statistical Manual of Mental Disorders (DSM)-IV criteria, medical history, urine test results and interview responses. The exclusion criteria included: DSM-IV criteria for an additional Axis I disorder; history of alcohol, cigarette, amphetamine, barbiturate, benzodiazepine or marijuana dependence according to DSM-IV; taking other prescribed medications that may affect the CNS; history of seizures, hematological diseases or severe liver or kidney impairment or pregnancy. A total of 205 healthy blood donors (mean age: $36.13 \pm 6.23$ years, 166 males, 39 females) were recruited at the First Hospital Affiliated to the Medical College of Xi'an Jiaotong University (Xi'an, China). Subjects who had substance abuse, participated in other studies or suffered from chronic brain diseases were excluded.

All participants were Han Chinese from Shaanxi (China) and not genetically related. Written informed consent was obtained from all participants. The study protocol was approved by the Ethical Committee of Xi'an Mental Health Center (Xi'an, China).

SNP selection and genotyping. SNPs in the promoter region, untranslated regions, exons, introns and 3 ' end of the RGS9 gene were systematically screened. The RGS9 gene with a genomic length of $1.1 \mathrm{Mb}$ and 10 selected SNPs selected for genotyping are shown in Table I. Preliminary analysis was performed using the HapMap data (http://www.ncbi.nlm. nih.gov/SNP/snp). Tag SNPs were examined with Haploview software version 4.2 (Broad Institute, Cambridge, MA, USA) using the Chinese Han in Beijing population and a minor allele frequency cut-off $\geq 5 \%$ (HapMap Data Release 27). The linkage disequilibrium (LD) pattern of this gene was determined in the Chinese population using the preliminary data from the HapMap. These SNPs were further analyzed in an association study.

Between 3-5 $\mathrm{ml}$ of peripheral blood were collected in tubes coated with EDTA. Genomic DNA was extracted from blood leukocytes using the EZNA ${ }^{\mathrm{TM}}$ Blood DNA Midi kit (Omega Bio-Tek, Norcross, GA, USA), according to the manufacturer's instructions. DNA was stored at $-80^{\circ} \mathrm{C}$ for SNP analysis. Cases and controls were mixed on the same plates and a double-blind procedure was performed. Probes and primers were designed using the Assay Design software version 3.4 (Sequenom Inc., San Diego, CA, USA) (Table II). SNP genotyping was performed using matrix assisted laser desorption ionization-time of flight mass spectrometry (MassARRAY system; Sequenom Inc.). Genotype calling was performed in real time using the MassARRAY RT software version 3.0.0.4 and data analysis was performed using the MassARRAY Typer software (version 3.4; Sequenom Inc.).

Statistical analysis. All statistical tests were conducted with SPSS 16.0 for Windows (SPSS, Inc., Chicago, IL, USA). The Hardy-Weinberg equilibrium (HWE) for each SNP was assessed using the Genepop v4.0 (19). Associations between the case-control status and each polymorphism were assessed by Fisher's exact test or Pearson's $\chi^{2}$ test. The odds ratio (OR) and $95 \%$ confidence interval (CI) were used to measure the strength of the association between allele frequencies and the RGS9 gene. The Bonferroni correction was used to adjust the test level when multiple comparisons were conducted and the P-value was divided by the total number of loci or haplotypes. Pair-wise LD statistics ( $\mathrm{D}^{\prime}$ and $\mathrm{r}^{2}$ ) and haplotype frequencies were computed using Haploview 4.0 to construct haplotype blocks. All $\mathrm{P}$ values presented were two sided, and $\mathrm{P}<0.05$ was considered to indicate a statistically significant difference.

\section{Results}

The distribution frequencies of the 10 genotyped SNPs were in agreement with the HWE. The analysis of linkage disequilibrium in the heroin-dependent patients and the healthy controls revealed that two SNPs were located in haplotype block 1 (rs1530351 and rs4791230), four SNPs were located in haplotype block 2 (rs8077696, rs8070231, rs2869577 and rs2292593) and four SNPs were located in haplotype block 3 (rs2292592, rs9916525, rs1122079 and rs4790953; D'>0.9; Fig. 1). The genotype distribution, allelic frequencies and haplotypes in the heroin-dependent patients and the healthy controls are shown in Tables I and III-V.

The results demonstrated that there were significant differences in the genotype frequency and allele frequency distribution of two SNPs (rs1530351 and rs4791230) between the heroin-dependent patients and the healthy controls. The frequency of the rs1530351 C allele was significantly higher in the heroin-dependent patients than in the healthy controls $\left(\chi^{2}=8.031, \mathrm{P}=0.005, \mathrm{OR}=2.079,95 \% \mathrm{CI}=1.241-3.483\right)$. Compared with the healthy controls, the heroin-dependent patients carried a higher frequency of the rs4791230G allele $\left(\chi^{2}=7.360, \mathrm{P}=0.007, \mathrm{OR}=2.021,95 \% \mathrm{CI}=1.205-3.389\right)$, whereas the remaining eight RGS9 SNPs exhibited negative results (Table I).

Furthermore, LD was observed in three blocks $\left(\mathrm{D}^{\prime}>0.9\right)$ and significantly less T-A haplotypes $\left(\chi^{2}=4.867, \mathrm{P}=0.027\right.$, $\mathrm{OR}=0.442,95 \% \mathrm{CI}=0.210-0.929)$ were identified in the heroin-dependent patients, suggesting that they may exhibit 


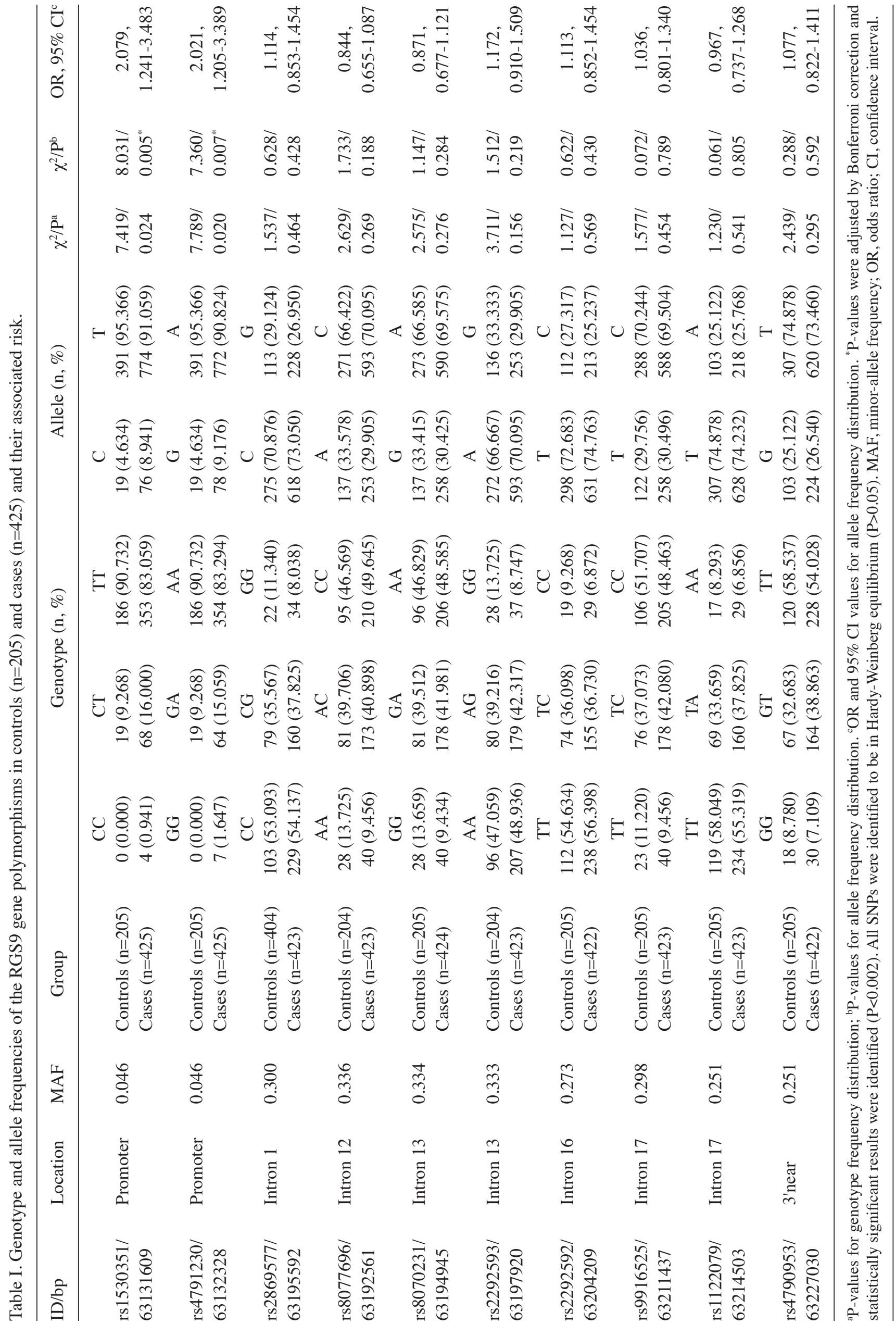




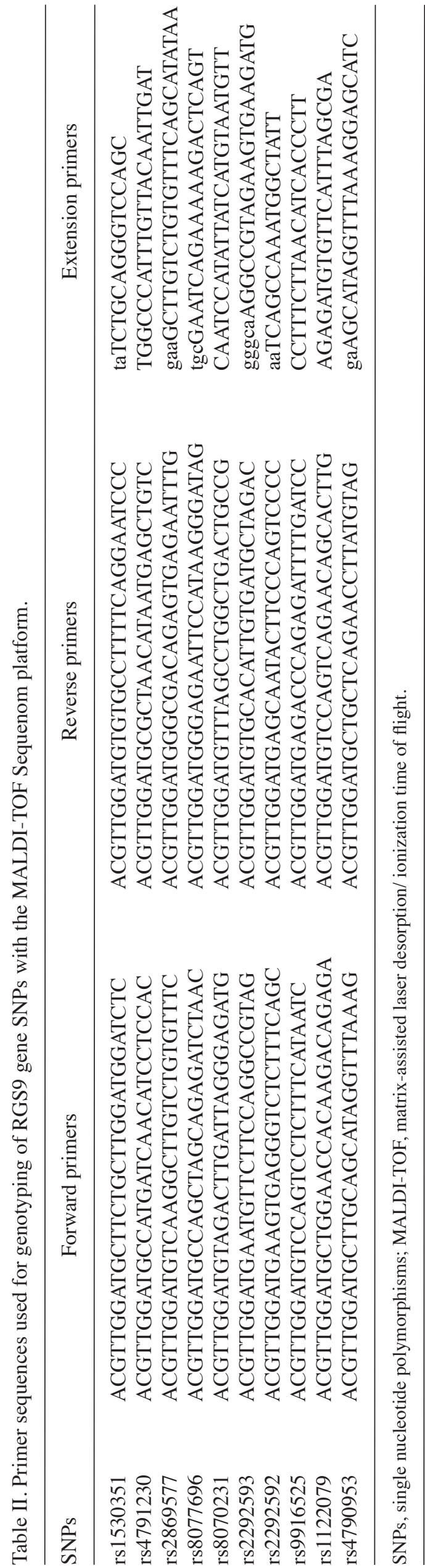

protective effects against heroin dependence, however, it did not pass the threshold value $(\mathrm{P}=0.025)$.

\section{Discussion}

RGS9 is a member of the RGS family of proteins and is specifically expressed in the striatum, which is involved in movement, motivation, mood and dependence $(14,20)$. Previous studies have demonstrated that acute morphine administration increases the expression of RGS9-2 in the nucleus accumbens and other CNS regions, whereas chronic administration decreases its expression (12-14). Mice lacking RGS9 exhibit enhanced behavioral responses to acute and chronic morphine administration, including a significant increase in morphine reward, increased morphine analgesia with delayed tolerance and exacerbated morphine physical dependence and withdrawal (6). This suggests that RGS9 acts upon the reward system of the brain, which is important in opiate dependence. Accumulated evidence indicates that RGS9 receptors are pivotal in the development of tolerance and physical dependence to opiates (6). These findings establish RGS9 as a potent negative modulator of opiate action in vivo and suggest that opiate-induced alterations in the level of RGS9 contribute to the behavioral and neural plasticity associated with chronic opiate administration (6). To the best of our knowledge, the present results provide the first direct evidence that a genetic change in the RGS9 gene is associated with heroin dependence in humans.

In the present study, significant differences were identified in the genotype frequency and allele frequency distribution of two SNPs (rs1530351 and rs4791230) in the promoter region between the heroin-dependent patients and the healthy controls. It was found that one SNP (rs1530351) in the RGS9 gene was significantly associated with increased risk of heroin dependence and another SNP (rs4791230) was also associated with increased risk of heroin dependence. To the best of our knowledge, this is the first study demonstrating a significant association between the RGS9 rs1530351 and rs4791230 SNPs with heroin dependence. A study confirmed that SNPs in the promoter region could alter microRNA binding to the promoter, resulting in the regulation of mRNA expression (21). These studies suggested that the RGS9 gene may contribute to the susceptibility to psychotic diseases. The SNPs in the promoter of the RGS9 gene affected mRNA stability and regulated mRNA expression (21), thereby affecting the genetic susceptibility to heroin dependence. Nevertheless, the role of the two SNPs in the genetic susceptibility to heroin dependence requires further investigation.

The genetic interactions among polymorphisms was further investigated and strong LD was observed. Haplotype analysis revealed that significantly less T-A haplotypes were identified in the heroin-dependent patients, although it did not pass the threshold value $(\mathrm{P}=0.025)$. There were significant point-wise associations of these variants with heroin dependence. These results indicated that the patients with T-A haplotypes of the RGS9 gene were less prone to heroin dependence, suggesting that they may exhibit protective effects against heroin dependence. Further studies are required to examine the protective effect of the T-A haplotypes of the RGS9 gene on the risk of heroin dependence. 
Table III. RGS9 haplotypes in block 1 frequencies and the results of their associations with the risk of heroin dependence.

\begin{tabular}{|c|c|c|c|c|c|c|}
\hline \multirow[b]{2}{*}{ Haplotype $^{\mathrm{a}}$} & \multirow[b]{2}{*}{ Cases $(\mathrm{n}, \%)$} & \multirow[b]{2}{*}{ Controls $(\mathrm{n}, \%)$} & \multicolumn{4}{|c|}{ Statistics } \\
\hline & & & $\chi^{2}$ & $\mathrm{P}^{\mathrm{b}}$ & OR & $95 \% \mathrm{CI}$ \\
\hline T-A & 385 (90.59) & $196(95.61)$ & 4.867 & 0.027 & 0.442 & $0.210-0.929$ \\
\hline C-G & 37 (8.71) & $10(4.88)$ & 2.935 & 0.087 & 1.860 & $0.906-3.818$ \\
\hline
\end{tabular}

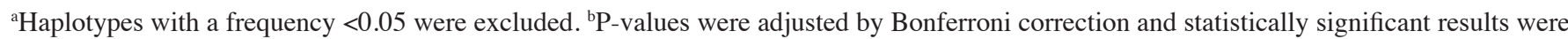
identified $(\mathrm{P}<0.025)$. OR, odds ratio; $\mathrm{CI}$, confidence interval.

Table IV. RGS9 haplotypes in block 2 frequencies and the results of their associations with the risk of heroin dependence.

\begin{tabular}{|c|c|c|c|c|c|c|}
\hline \multirow[b]{2}{*}{ Haplotype $^{a}$} & \multirow[b]{2}{*}{ Cases $(\mathrm{n}, \%)$} & \multirow[b]{2}{*}{ Controls (n, \%) } & \multicolumn{4}{|c|}{ Statistics } \\
\hline & & & $\chi^{2}$ & $\mathrm{P}^{\mathrm{b}}$ & OR & $95 \% \mathrm{CI}$ \\
\hline C-A-C-A & $292(68.71)$ & 132 (64.39) & 1.170 & 0.279 & 1.214 & $0.854-1.726$ \\
\hline A-G-G-G & $108(25.41)$ & $57(27.80)$ & 0.410 & 0.522 & 0.885 & $0.608-1.288$ \\
\hline A-G-C-G & $16(3.76)$ & $11(5.37)$ & 0.864 & 0.353 & 0.690 & $0.314-1.515$ \\
\hline
\end{tabular}

${ }^{\text {a}}$ Haplotypes with a frequency $<0.05$ were excluded. ${ }^{b} \mathrm{P}$-values were adjusted by Bonferroni correction and statistically significant results were identified $(\mathrm{P}<0.0125)$. OR, odds ratio; $\mathrm{CI}$, confidence interval.

Table V. RGS9 haplotypes in block 3 frequencies and the results of their associations with the risk of heroin dependence.

\begin{tabular}{|c|c|c|c|c|c|c|}
\hline \multirow[b]{2}{*}{ Haplotype $^{\mathrm{a}}$} & \multirow[b]{2}{*}{ Cases $(\mathrm{n}, \%)$} & \multirow[b]{2}{*}{ Controls $(\mathrm{n}, \%)$} & \multicolumn{4}{|c|}{ Statistics } \\
\hline & & & $\chi^{2}$ & $\mathrm{P}^{\mathrm{b}}$ & OR & $95 \% \mathrm{CI}$ \\
\hline T-C-T-T & $288(67.76)$ & $139(67.80)$ & 0.000 & 0.992 & 0.998 & $0.699-1.426$ \\
\hline C-T-A-G & $102(24.00)$ & $50(24.39)$ & 0.012 & 0.915 & 0.979 & $0.664-1.444$ \\
\hline T-T-T-T & $20(4.71)$ & $9(4.39)$ & 0.096 & 0.764 & 1.131 & $0.506-2.531$ \\
\hline
\end{tabular}

${ }^{\mathrm{a}}$ Haplotypes with a frequency $<0.05$ were excluded. ${ }^{\mathrm{b}} \mathrm{P}$-values were adjusted by Bonferroni correction and statistically significant results were identified $(\mathrm{P}<0.0125)$. OR, odds ratio; $\mathrm{CI}$, confidence interval.

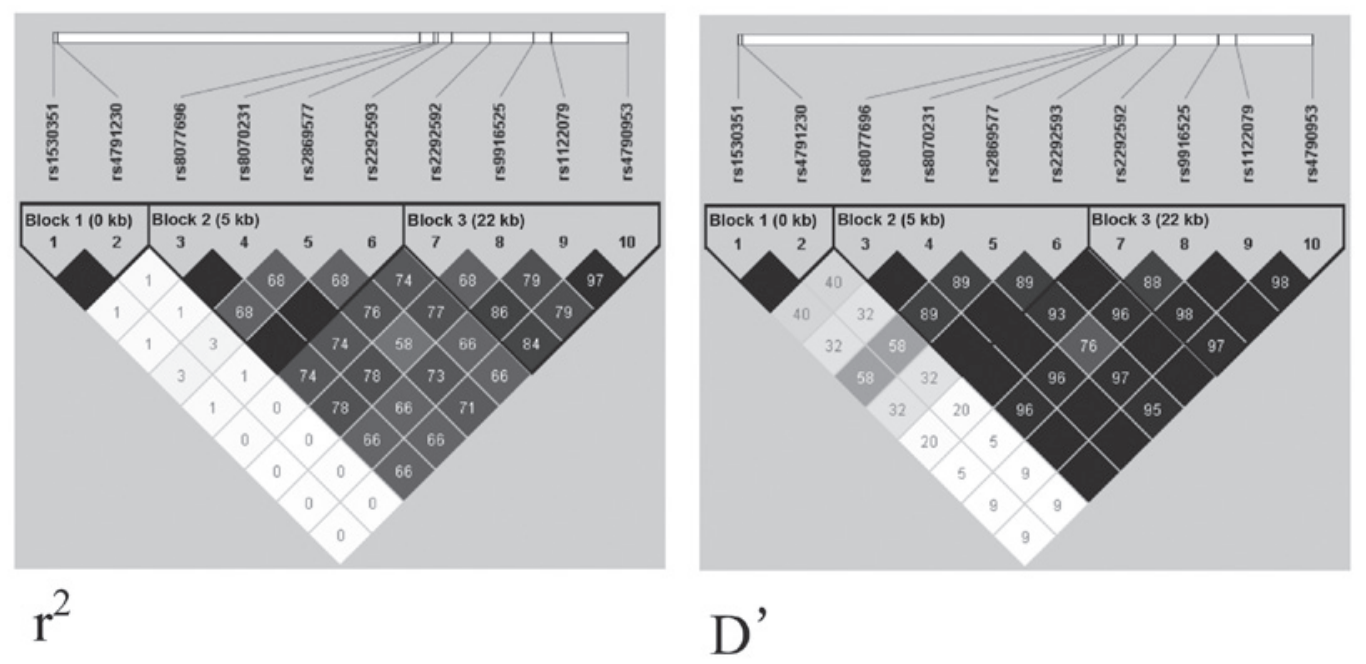

Figure 1. LD plot of the 10 SNPs in the regulator of G protein signaling 9 gene. Values in squares are the pair-wise calculation of $\mathrm{r}^{2}$ (left) or D' (right). Black squares indicate $r^{2}=1$ (i.e. perfect LD between a pair of SNPs). Empty squares indicate $D^{\prime}=1$ (i.e. complete LD between a pair of SNPs). LD, linkage disequilibrium; SNPs, single nucleotide polymorphisms. 
In conclusion, the present study identified a strong association between two SNPs (rs1530351 and rs4791230) in the RGS9 gene and heroin dependence. These findings encourage future efforts in searching for functional polymorphisms within and close to the RGS9 gene using a systemic approach in a larger sample population.

\section{Acknowledgements}

This study was supported by the Research and Development Foundation of Science and Technology of Shaanxi Province grant no. 2012K16-03-01) and the National Natural Science Foundation of China (grant no. NSFC31100900).

\section{References}

1. Koob GF: The neurobiology of addiction: a neuroadaptational view relevant for diagnosis. Addiction 101 (Suppl 1): 23-30, 2006.

2. Kreek MJ, Nielsen DA, Butelman ER and LaForge KS: Genetic influences on impulsivity, risk taking, stress responsivity and vulnerability to drug abuse and addiction. Nat Neurosci 8: $1450-1457,2005$.

3. Cadoret RJ, Yates WR, Troughton E, Woodworth G and Stewart MA: Adoption study demonstrating two genetic pathways to drug abuse. Arch Gen Psychiatry 52: 42-52, 1995.

4. Uhl GR: Molecular genetics of substance abuse vulnerability: remarkable recent convergence of genome scan results. Ann NY Acad Sci 1025: 1-13, 2004.

5. Uhl GR, Drgon T, Johnson C, et al: 'Higher order' addiction molecular genetics: convergent data from genome-wide association in humans and mice. Biochem Pharmacol 75: 98-111, 2008

6. Zachariou V, Georgescu D, Sanchez N, et al: Essential role for RGS9 in opiate action. Proc Nal Acad Sci USA 100: 13656-13661, 2003.

7. Badgaiyan RD: A novel perspective on dopaminergic processing of human addiction. J Alcohol Drug Depend 1: 1000e101, 2013.
8. Volkow ND, Fowler JS, Wang GJ, Swanson JM and Telang F: Dopamine in drug abuse and addiction: results of imaging studies and treatment implications. Arch Neurol 64: 1575-1579, 2007.

9. Dohlman HG and Thorner J: RGS proteins and signaling by heterotrimeric G proteins. J Biol Chem 272: 3871-3874, 1997.

10. Rahman Z, Gold SJ, Potenza MN, et al: Cloning and characterization of RGS9-2: a striatal-enriched alternatively spliced product of the RGS9 gene. J Neurosci 19: 2016-2026, 1999.

11. Birnbaumer L: Expansion of signal transduction by $\mathrm{G}$ proteins. The second 15 years or so: from 3 to 16 alpha subunits plus betagamma dimers. Biochim Biophys Acta 1768: 772-793, 2007.

12. Traynor JR and Neubig RR: Regulators of G protein signaling and drugs of abuse. Mol Interv 5: 30-41, 2005.

13. Hooks SB, Martemyanov K and Zachariou V: A role of RGS proteins in drug addiction. Biochem Pharmacol 75: 76-84, 2008.

14. Traynor JR, Terzi D, Caldarone BJ and Zachariou V: RGS9-2: probing an intracellular modulator of behavior as a drug target. Trends Pharmacol Sci 30: 105-111, 2009.

15. Zhang K, Howes KA, He W, et al: Structure, alternative splicing and expression of the human RGS9 gene. Gene 240: 23-34, 1999.

16. Escamilla M, Hare E, Dassori AM, et al: A schizophrenia gene locus on chromosome 17q21 in a new set of families of Mexican and central american ancestry: evidence from the NIMH genetics of schizophrenia in latino populations study. Am J Psychiatry 166: 442-449, 2009.

17. Greenbaum L, Pelov I, Teltsh O, Lerer B and Kohn Y: No association between regulator of G-protein signaling 9 (RGS9) and schizophrenia in a Jewish population. Psychiatr Genet 20: 47-48, 2010.

18. Clarke GM Anderson CA, Pettersson FH, Cardon LR, Morris AP and Zondervan KT: Basic statistical analysis in genetic case-control studies. Nat Protoc 6: 121-133, 2011.

19. Rousset F and Raymond M: Testing heterozygote excess and deficiency. Genetics 140: 1413-1419, 1995.

20. Anderson GR, Posokhova E and Martemyanov KA: The R7 RGS protein family: multi-subunit regulators of neuronal $\mathrm{G}$ protein signaling. Cell Biochem Biophys 54: 33-46, 2009.

21. Zhou Y, Shi T, Mozola MA, et al: Evidence that the promoter can influence assembly of antitermination complexes at downstream RNA sites. J Bacteriol 188: 2222-2232, 2006. 